\title{
Advanced Metering and Demand Response Communication Performance in Zigbee based HANs
}

\author{
Velin Kounev \\ School of Information Science \\ University of Pittsburgh \\ Pittsburgh, PA 15260 \\ Email: vkounev@pitt.edu
}

\author{
David Tipper \\ School of Information Science \\ University of Pittsburgh \\ Pittsburgh, PA 15260 \\ Email: dtipper@pitt.edu
}

\begin{abstract}
Using IEEE 802.15.4 and Zigbee for home area networks (HANs) in the Smart Grid is becoming an increasingly prominent topic in the research area. As the standard designed for low data rate and low cost wireless personal area networks, IEEE 802.15.4 is widely employed in the construction of home sensor networks to assist with real-time environment information. For the purposes of Smart Grid the Zigbee Alliance has defined new Smart Energy Profile Protocol that leverages the existing TCP and HTTP protocols. In this paper, we provide an overview of the Smart Grid's Advanced Metering Infrastructure (AMI) and Demand Response (DR) functionalities, and the communication requirement they pose for the new SEP protocol. The discussion is followed by an evaluation of the theoretical performance bounds of the new architecture based on a analytical model. We conclude, by extending the model to account for WiFi interference which is expected to be present in home and office environments.
\end{abstract}

\section{INTRODUCTION}

Number of independently own and operated power plants and transmission lines is what today constitutes the U.S. electric grid. This complexity of the network coupled with aging infrastructure and ever increasing power consumption, has forced the experts to examine the status and health of the system. The grid includes the wires, transformers, switches and substation that carry the electricity from the power plants to the customers. One of the main drawback is the time and money spend on repairs. Utility companies have to send out workers to gather most of the information needed to provide electricity, such as read meters, look for broken equipment and measure voltage. Currently many options and products are being made available to the electrical industry to modernize it [1]-[3]. The vision is that fully automated information and process control system would provide improved performance, by automated information gathering and assistance prior to any repairs.

By using computer based remote control and automation systems, the current electrical grid is envisioned to be evolved into "Smart Grid". This is made possible by two-way communication technology and computer processing. Great improvements in energy efficiency, isolation of electrical failures and short recovery times are few advantages to customers and utilities.

\section{A. Smart Grid Application Requirments}

The U.S. Department of Energy (DOE), in collaboration with the Federal Energy Regulatory Commission (FERC) and the National Institute of Standards and Technology (NIST), sets forth six key functionalities to be achieved by Smart Grid Infrastructure: (1) Advanced Metering Infrastructure; (2) Demand Response; (3) Electric Vehicles; (4) Wide-area Situation Awareness; (5) Distributed Energy Resources and Storage; and, (6) Distribution Automation [4].

Each set of functions has its own requirements in terms of bandwidth, latency and availability for the communication systems. The communication infrastructure is divided into 3 parts: Home Area Network, Neighborhood Area Network and Wide Area Network. Summaries and network allocation for each Smart Grid functionality is presented below.

1) Advanced Metering Infrastructure (AMI): An advanced two-way communication system to collect and measure energy consumption for billing and statistical purposes. Future AMI evolutions envision near-real-time operations. Requirements such as bandwidth, availability and latency are expected to grow to significantly higher level.

2) Demand Response (DR): One of the most significant advantages of Smart Grid conceived to reduce peak loads. The final most advance version of DR is automated DR, allowing on-premise smart appliances to respond to dynamic condition on the grid, and shift load consumption in a near-real-time manner.

3) Wide Area Situational Awareness (WASA): A set of technologies designed to improve the monitoring of the power system across large geographical areas, and in the event of emergency to isolate failures from spreading to the rest of the network. A disturbance in the power supply in one area can quickly spread and become a widespread problem, with cascading and deleterious consequences [4]

4) Distributed Energy Resources and Storage (DER): In Smart Grid the energy flow will be multi-directional, from utility to home, home to utility, or from home to home. The real-time net metering is required as to measure the electricity drawn from the grid minus the energy provided by energy sources on the premises.

5) Electric Vehicles $(E V)$ : A more robust grid is needed, due to the fact that the current electrical grid is unlikely to provide the peak capacity required to charge a significant number of EV during peak hours.

6) Distribution Automation (DA): The primary function of DA is to reduce voltage to an appropriate level in order to isolate potential faults. Furthermore, the utilities would be able to remotely monitor and control on-premise equipment through automated decision-making. 


\begin{tabular}{|c|c|c|}
\hline Communication Network & Smart Grid Functionality & Bandwidth, Latency, Availability \\
\hline \multirow[t]{2}{*}{ Home Area Network (HAN) } & & (Most Stringent: $300 \mathrm{kbps} /$ node, 0.02 seconds, 99.99\%) \\
\hline & $\begin{array}{l}\text { Advanced Metering Interface (AMI) } \\
\text { Demand Response (DR) } \\
\text { Distributed Energy Resources and Storage (DER) } \\
\text { Electric Vehicles (EV) }\end{array}$ & $\begin{array}{l}100 \mathrm{kbps} / \text { node, } 2-15 \mathrm{sec}, 99-99.99 \% \\
100 \mathrm{kbps} / \text { node, } 0.5-2 \mathrm{sec}, 99-99.99 \% \\
100 \mathrm{kbps} / \text { node, } 0.02-15 \mathrm{sec}, 99-99.99 \% \\
100 \mathrm{kbps} / \text { vehicle, } 2 \mathrm{sec}-5 \mathrm{~min}, 99-99.99 \%\end{array}$ \\
\hline \multirow{2}{*}{ Neighborhood Area Network (NAN) } & & (Most Stringent: $2 \mathrm{Mbps} /$ home, 0.02 seconds, 99.999\%) \\
\hline & $\begin{array}{l}\text { Advanced Metering Interface (AMI) } \\
\text { Demand Response (DR) } \\
\text { Distributed Energy Resources and Storage (DER) } \\
\text { Electric Vehicles (EV) } \\
\text { Distribution Automation (DA) }\end{array}$ & $\begin{array}{l}500 \mathrm{kbps} / \text { node, } 2-15 \mathrm{sec}, 99-99.99 \% \\
500 \mathrm{kbps} / \text { node, } 2 \mathrm{sec}, 99-99.99 \% \\
500 \mathrm{kbps} / \text { node, } 0.3-15 \mathrm{sec}, 99-99.99 \% \\
100 \mathrm{kbps} / \text { vehicle, } 2 \mathrm{sec}-5 \mathrm{~min}, 99-99.99 \% \\
100 \mathrm{kbps} / \text { vehicle, } 0.1 \mathrm{sec}-1 \mathrm{sec}, 99-99.999 \%\end{array}$ \\
\hline \multirow[t]{2}{*}{ Wide Area Network (WAN) } & & (Most stringent: Gbps (variable), 0.02 seconds, 99.999\%) \\
\hline & $\begin{array}{l}\text { Advanced Metering Interface (AMI) } \\
\text { Demand Response (DR) } \\
\text { Distributed Energy Resources and Storage (DER) } \\
\text { Wide Area Situational Awareness (WASA) } \\
\text { Electric Vehicles (EV) } \\
\text { Distribution Automation (DA) }\end{array}$ & $\begin{array}{l}500 \mathrm{kbps} / \text { node, } 2-15 \mathrm{sec}, 99-99.99 \% \\
500 \mathrm{kbps} / \text { node, } 2 \mathrm{sec}, 99-99.99 \% \\
500 \mathrm{kbps} / \text { node, } 0.3-15 \mathrm{sec}, 99-99.99 \% \\
600-1500 \mathrm{kbps} / \text { node, } 0.02-0.2 \mathrm{sec}, 99.999 \% \\
100 \mathrm{kbps} / \text { vehicle, } 2 \mathrm{sec}-5 \mathrm{~min}, 99-99.99 \% \\
100 \mathrm{kbps} / \text { vehicle, } 0.1 \mathrm{sec}-1 \mathrm{sec}, 99-99.999 \%\end{array}$ \\
\hline
\end{tabular}

TABLE I

SUMMARY OF SMART GRID FUNCTIONS AND THEIR COMMUNICATION NETWORK ASSIGNMENTS. FOR EACH NETWORK TOTAL FOR BANDWIDTH, LATENCY AND AVAILABILITY REQUIREMENTS ARE PRESENTED, WITH MOST STRINGENT REQUIRMENTS LISTED (AS INDICATED BY THE DEPARTMENT OF ENERGY [4]).

\section{B. Smart Grid Communication Networks}

Four of the functions of Smart Grid span over all three communication networks. Advanced Metering Infrastructure, Demand Response, Distributed Energy Resources and Storage, and Electric Vehicle would have communication needs in the entire Smart Grid network. A summary of all Smart Grid functions and their assignments are presented in Table I. For each telecommunication network section, the total bandwidth, latency and availability requirements are presented. Please note, that for each communication network requirement, the most stringent requirement is selected [4].

\section{Home Area Network Communication Technologies}

Home Area Network is the last hop of next-generation Smart Grid networks [5]. The design envisions that the network shall be a one-hop network from each individual smart appliance to a concentrator device. This device shall be in charge of collecting the home information and sending it to the utilities. The concentrator device is commonly referred as a Smart Meter, and it is already available for purchasing. In general, smart meters come equipped with at least two network interfaces - one or more for inside communication and one for outside communication. The outside interface connects the Smart Meter to the Neighborhood Area Network (NAN), and the inside one is used for connection to all smart appliances. NAN communication network is outside the scope of this paper.

There are two types of communication architectures envisioned to be used as last hop for Smart Grid: Power Line Communication (PLC) and wireless transmission technologies. PLC makes use of existing in-house power line infrastructure to transmit data and control signals. The main advantage of those technologies is that power lines have extended to every residence with multiple outlets installed in each room. The candidates protocols for HAN communication via PLC are
$\mathrm{X}-10$, Insteon, PLC-BUS, LonWorks and HomePlug. While the industry had not converged, the predominant technology used in installation today is Zigbee, followed by HomePlug [4]. In North America, wireless is the dominant technology of choice for HAN while in Europe PLC is the leading technology. According to study conducted by General Electric, WiFi $(802.11 \mathrm{n})$ and Zigbee are the two technologies that best meet HAN requirements [6]. One of the main reason for that being cost effectiveness, and flexibility in contrast to any wired based technology.

The remainder of this paper is organized as follows: Section II gives an overview of Zigbee in regards to Smart Grid, including Smart Energy Profile Protocol. Section III presents a theoretical model for calculating the performance of Zigbee's SEP protocol in regards to AMI and DR functions. Finally, theoretical results are shown in Section IV. Our conclusion is drawn in section $\mathrm{V}$.

\section{Zigbee IN Home Area Network}

\section{A. IEEE802.15.4 and Zigbee Relatioship}

IEEE 802.15.4 is part of the IEEE family of standards for physical and link-layers for wireless personal area networks (WPANs). The WPAN working group focuses on short range wireless links, in contrast to local area and metropolitan area coverage explored in WLAN and WMAN working groups, respectively. The focus area of IEEE 802.15.4 is that of low data rate WPANs, with low complexity and stringent power consumption requirements. The standard is designed to be a cost effective, low-power, and low-interference technology. The technology operates on the same $2.4 \mathrm{GHz}$ ISM band as WiFi, Bluetooth and WiMax [7]. Depending on the environment and the power used for transmission, IEEE 802.15.4 compliant wireless devices are expected to transmit in a range of 10 to 75 meters [8].

The Zigbee Alliance is a group of over 400 member companies that maintain and publish technical standards. Zigbee is a 
registered trademark of Zigbee Alliance, and the relationship between IEEE 802.15.4 and the group is similar to that of IEEE 802.11 and WiFi Alliance. IEEE defines the physical and medium access layers while the Zigbee Alliance defines network and application layers. The group recognizes that the co-existence of different wireless technologies on the same frequencies can have a significant impact on network operations.

\section{B. Zigbee Modes of Operation}

The channel access mode in IEEE 802.15.4 is carrier sense, multiple access with collision avoidance (CSMA/CA) [8]. The network can operate in beacon-enable and nonbeacon-enabled modes. Those modes allow the end devices to choose their sleep patterns. In non-beacon-enable mode, devices simply transmit data frames using un-slotted CSMA/CA to the coordinator [8]. Each device must perform two clear channel assessments (CCA) prior to transmitting. The two CCA operations ensure prevention of potential collisions. Each time the channel is assessed as busy via CCA, the device must back off for a random duration. If the number of back-offs reaches the maximum, by default set to 5 , the high layer application is informed of communication failure. Furthermore, in CSMA/CA mode IEEE 802.15.4 supports optional retransmission scheme based on acknowledgements [9]. When retransmissions are enabled, the receiver node must send a positive acknowledgement right after receiving a data frame. If the acknowledgment is not (correctly) received by the sender, a retransmission is started unless the maximum number of retransmissions is reached. In this case, the data frame is dropped. The maximum retransmission retries can be set between 0 and 7 with a default value of 3 . The beacon interval/superframe equals to 960 symbols. Depending on parameters, the range between beacons in $2.4 \mathrm{GHz}$ Zigbee varies between $15.35 \mathrm{~ms}$ to $251.7 \mathrm{~s}$ [10]. The active portion of the superframe is divided into 16 slots. The default value of each slot is 60 symbols, with 4 bits per symbol and 0.96 ms per time slot in the $2.4 \mathrm{GHz}$ range. There are three parts in the active portion: a beacon, a contention access period (CAP), and a contention-free period (CFP). In the CAP, all data transmissions shall follow a successful execution of a slotted CSMA/CA algorithm [22]. The algorithm is the same as un-slotted CSMA/CA, with the exception that each operation - channel access, back-off count and clear channel assessment, can only begin at the boundaries of a basic time unit called Backoff Period (BP). This period is equal to 80 bits or $0.32 \mathrm{~ms}[10]$.The $\mathrm{BP}$ must also align with the beginning of each superframe. As in un-slotted CSMA/CA, nodes may transmit after two consecutive successful CCA. For lowlatency applications, upon request from a node, the coordinator may assign guaranteed time slots to an end device during the guaranteed time slot (GTS) period. There are a maximum of seven GTSs in a superframe, and time slots are allowed to occupy more than one GTP. Prior to transmission the node must ensure there is sufficient time left in the GAP for the transmission and any consecutive acknowledgments packets, if positive feedback option is selected. Multiple data frames may be sent within a single time slot. The guaranteed portion of the frame can dynamically shrink or grow; however, a minimum of 440 symbols, or $7 \mathrm{~ms}$, is reserved for the contention period, for contention-based access of other networked devices or new devices wishing to join the network. Due to the time-sensitive nature of Smart Grid, GTS mode in beacon enabled mode is assumed to be used.

\section{Smart Energy Profile Protocol}

In context of Smart Grid, appliances would only implement 4 network layers [11]. Contrary, to the classic OSI model, transport, session and presentation layers are not present. The HomePlug Alliance, Wi-Fi Alliance, HomeGrid Forum and Zigbee Alliance have agreed to create a consortium for interoperability. In July of 2012, the alliances published a new draft for Smart Grid application communication as Smart Energy Profile 2.0 Application Protocol Specification [12] [13]. The purpose of the documents is to define the interface and messages between smart appliances, smart meters and utilities, to be used by layer 4 Smart Grid applications. The protocol is defined as HTTPS over TCP based pull mechanism with the assumption that the pull subscription/notification mechanism may not be reliable. The TCP protocol is introduced as part of the application layer. The basic operations in the protocol are post, put and delete commands. Furthermore, devices are expected to have intermediate connection to the network due to sleep cycles. The draft states "to prevent overwhelming network resources, notifications SHOULD be sent to a given client for a given resource no more than once every 30 seconds. Notifications for conditional subscriptions SHOULD only be sent once within this time period for a given client for a given resource and any additional notifications SHOULD NOT be queued. All devices need to be considerate of network resources." [12]. Additionally, the end devices are responsible for pulling network time from the network controller; however, the granularity of the device time shall be 1 second. By default, smart appliances will have duty cycles of $90 \%$, meaning that for every 30 minutes, the devices shall sleep for 3 minutes. These specifications directly contradict with the requirements published by the Department of Energy [4], in regards to Advanced Metering Interface (AMI) and Demand Response (DR) and Distributed Energy Resources and Storage (DER) applications in home area network (refer to Table I). Table II presents minimum and maximum Smart Energy Profile protocol data and control information lengths (defined in [13]). The message size summary considers all 4 Zigbee layers, and it includes header size for mandatory and optional HTTP headers (as defined in [12]), TCP and Zigbee headers.

As it can be see from the summary above, the protocol defined maximum and minimum lengths for each message portion, as well as mandatory or optional status. Using the information provided, we can compute the theoretical minimum and maximum for SEP protocol message. The minimum considers the lower bound of fields lengths and excludes all optional HTTP headers, resulting in total length of 508 bytes. By contrast, the maximum length considers all header field and their maximum lengths, resulting in 1524 bytes per SEP message. Those theoretical bounds would be used to calculate the delay statistics presented in the sections that follow.

\section{SEP Theoretical Performance in HAN}

\section{A. Performance in Ideal Conditions}

In [14], Koubaa et al. proposed a mathematical model to compute an upper bound on guaranteed data rate and 


\begin{tabular}{lll}
\hline Type & Length & SEP Status \\
\hline AMI \& DR Application & $256-1024$ bytes & Mandatory \\
HTTP Accept Header & $36-116$ bytes & Mandatory \\
HTTP Connection Header & $17-22$ bytes & Mandatory \\
HTTP Content-Length Header & $18-22$ bytes & Optional \\
HTTP Content-Range Header & $32-38$ bytes & Optional \\
HTTP Content-Type Header & $18-23$ bytes & Mandatory \\
HTTP Host Header & $30-100$ bytes & Mandatory \\
HTTP Location Header & $20-50$ bytes & Mandatory \\
TCP Header & 30 bytes & Mandatory \\
Zigbee Header & 15 bytes & Mandatory \\
\hline
\end{tabular}

TABLE II

SUMMARY OF SEP PAYLOAD AND CONTROL INFORMATION MESSAGES AND LENGTHS.

worst case latency in beacon-enabled GTS Zigbee networks. The authors argued that Zigbee networks are low jitter, and questioned the general assumption that network clients traffic is Poisson generated. They recommended the use of a network calculus $(b, r)$ model since machine-to-machine traffic is cyclical in nature. There are two parameters to the model: an arrival curve that upper bounds the actual traffic patterns, such that the received service at any time slice never exceeds the offered load; and minimum service curve guaranteed to approximate the actual network service rate. In Figure 1, the arrival curve (red line) is a function of maximum burst size $\mathrm{b}$, and average arrival rate $\mathrm{r}$, as to $\alpha(t)=b+r t$.

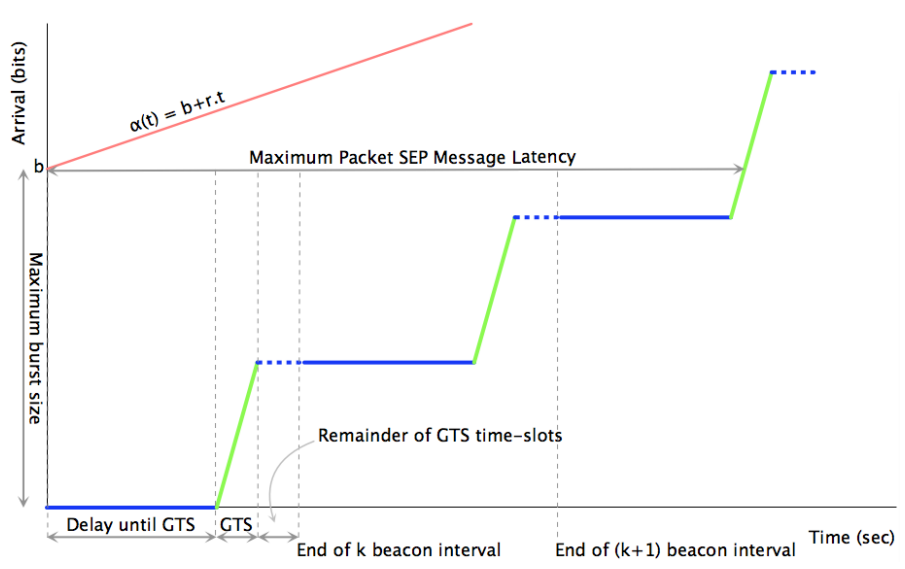

Fig. 1. The GTS (b,r)-based performance model.

The service process is presented by stair function that approximated IEEE 802.15.4 GTS service behavior, as

$$
B_{C, T}(t)=C * T_{G T S}+C *\left(t-\left(2 * B I-T_{\text {Reminder }}\right)\right)
$$

In the stair function, $\mathrm{C}$ (green lines) represents the service rate, which in the case of Zigbee is $250 \mathrm{kbps}$. During the
Beacon Interval (BI), $T_{G T S}$ is the time during which the client transmits data, and $T_{\text {Reminder }}$ is the left over from the clients GTS slot without any data transmission.

The total data rate of a client is summation of all Beacon Intervals: all data transmissions in the previous time slots ( $\mathrm{k}$ 1 ), plus data transmission in the current time slot (k), or

$$
B_{\text {stair }}=\sum B_{C, T}(t)
$$

The maximum horizontal distance $\left(D_{\max }\right)$ indicates the delay bound, occurring immediately after the initial burst period (the intersection of $\alpha(t)$ and the $\mathrm{y}$-axis). Depending on the transmission rate $\mathrm{C}$, the horizontal delay line may or may not intersect with the service curve during service cycle 1 (where $\mathrm{k}$ is the cycle index). As such, the initial burst may not get serviced until a number of cycles later. To illustrate in Figure 1, the initial burst gets serviced over the first 3 cycles. The maximum delay bound is expressed as

$D_{\text {max }}=\frac{b}{C}+(k+1) * B I-\left(T_{G T S}+T_{\text {Reminder }}\right)-k * T_{G T S}$

We proceed to calculate guaranteed bandwidth and delay bound for AMI and DR functions over SEP protocol in Smart Grid's HAN.

\section{B. Performance in Real World Conditions}

For the purposes of Smart Grid, $2.4 \mathrm{GHz}$ ISM band must be used. This is due to data rate requirements of around 300 kbps per node in home area networks (refer to Table I), and the data rate limitations of the alternative two band. This is the same frequency band as that used by the IEEE 802.11 standard. In North America, all but 2 Zigbee channels, 25 and 26, out of the 16 available are non-overlapping with WiFi channels. Further, channel 26 is not legal in number of locals and it is not supported by certain standards. In Europe, WiFi channels have a different assignment, and only Zigbee channels 15, 16, 21, 22 are non-overlapping with WiFi [15]. It is a safe assumption that Home Area Network in Smart Grid would experience interference from WiFi networks. Liang at al. [16] conducted a thorough investigation of Wi-Fi to Zigbee interference. The research was motivated by observations made during a large conference (well above 7,000 attendees), where the reachability of individual nodes in a Zigbee based sensor network dropped down to $40 \%$ at worst. Given that observation, an experiment was conducted with a pair of Zigbee sender and receiver nodes and a pair of $802.11 \mathrm{~b} / \mathrm{g}$ based interferers. The distance between the two networks was varied between 15 to 170 feet, in order to characterize the spacial effect. The authors found that in case of $802.11 \mathrm{~b}$ the ZigBee network packet loss/corruption varied between $99 \%$ at 15 feet to $30 \%$ at 170 feet. The authors noted that by using CSMA/CA, Zigbee was able to detect WiFi transmission and back-off as per protocol requirement. On the contrary, WiFi was not able to detect Zigbee transmission via CSMA/CA if the distance between the Zigbee transmitter and the WiFi receiver was more than few meters. The authors concluded this was due to the difference in transmission power between the two: $16 \mathrm{dBm}$ for Wi-Fi and $0 \mathrm{dBm}$ for Zigbee. As such, a large number of Zigbee packets were corrupted due to pre-emptive (non-detected Zigbee channel occupancy) WiFi transmission. 
Furthermore, in [17], Ghosh el al. conducted a large scale WiFi traffic investigation in public hot-spots and showed the probability of the number of WiFi clients present at any given time. The investigation concluded that, the probability of having at least one WiFi client was above $99.999 \%$, and the probability of having 10 or more clients was above $93 \%$. We can safely assume, that in during normal HAN operation in home or business setting, Zigbee based networks would have to overcome interference from WiFi networks.

To account for this fact, the theoretical performance model is further extended to include the effect of WiFi interference, taking into account the probability of Zigbee successfully accessing the channel. We can assume that WiFi traffic pattern are independent of Zigbee [18]. We can model the probability of transmission of Smart Appliance during it's assigned timeslot as product of the probability of sensing clear channel and number of allowed re-tries, or

$$
C_{a d j}=p * C^{\prime} * \sum_{i=0}^{4}(1-p)^{i}
$$

where $p$ is the probability of Clear Channel Assessment and $C^{\prime}$ the reminder of the GTS bandwidth. The equation captures Zigbee's protocol specification, as the node performs a backoff after each unsuccessful transmission attempt. The backoff interval is uniformly distributed and there is a maximum of 5 re-transmission attempts before channel access failure is declared.

\section{Model Results}

Using the model presented in the prior section, we compute theoretical SEP performance over Zigbee.

\begin{tabular}{ll}
\hline Parameter & Value \\
\hline Duty Cycle & 1 \\
$\lambda$ & $1 / 16$ \\
Service Rate & $250 \mathrm{Kbps}$ \\
Protocol Overhead & $12 \%$ \\
Beacon Duration & 960 symbols (480 bytes) \\
Superframe interval & 0.00192 sec \\
CAP size & 440 symbols (4 bits/symbol) \\
Burst size & $508-1524$ bytes \\
Prob. of Clear Channel & $0.1-99 \%$ \\
GTS allocation & $1-7$ \\
\hline & TABLE III \\
SUMMARY OF PARAMERES USED IN MODEL COMPUTATION.
\end{tabular}

Table III presents the parameters used in the computation. It is assumed that in the context of Smart Grid, appliances would have sufficient power and therefore would not have a sleep cycle, resulting in a duty cycle of 1 . This means that the entire portion of the Zigbee frame would be active and used for communication. Since AMI and DR function are delay sensitive, we assume that the minimum Superframe duration is used, which results in Beacon Interval of 960 symbols, or $1.92 \mathrm{~ms}$ at $250 \mathrm{Kbps}$. Furthermore, minimum CAP interval per protocol requirement is assumed, and each smart appliance is assigned one or more GTS. The SEP protocol message size has a minimum of 508 and maximum of 1524 bytes (Table II). This is the length of one message, however due to the SEP PULL mechanism design, each AMI or DR message would require two transaction - one to query the smart meter, and one response from the smart meter. One GTS slot is assumed to take up to 1/16th of the Superframe duration and the Zigbee protocol overhead is set at $12 \%$ (consistent with [14]). The results presented in this section, do not include any processing time, or further delay in the rest of the Smart Grid Network. The only delay presented is the last hop delay experienced during the final information handoff between the Smart Meter and the smart appliances. In Figure 2, the theoretical delay and guaranteed throughput of the SEP protocol over Zigbee wireless links are presented as a function of the number of GTS assignment per device. As indicated previously, Zigbee can support up to 7 GTS clients, or 1 client with a maximum of 7 combined GTS. As expected, the guaranteed throughput has linear relationship to the number of GTS time-slots assigned. The minimum value is around $13.5 \mathrm{Kbps}$, and the maximum is around $95 \mathrm{Kbps}$. The delay experienced, on the other hand, is not linear and has a significant drop as the number of GTS assigned slots increase. These results are somehow expected, since with a single assigned time-slot the client has to wait for most of the Superframe duration before transmitting. As the number of assigned slots increases, the client spends less time waiting and more time transmitting, thus achieving, higher combined throughput and resulting in lower delay. After 3 or more GTS time-slots the delay is not reduced significantly, since most of the SEP message data can be transmitted in one or two cycles. From the results, we can conclude that with 1 assigned GTS time-slot, a SEP message would experience delay between 1.10 and $3.35 \mathrm{sec}$; however, as for 3 time-slots the delay drops to 0.1 and $0.034 \mathrm{sec}$. If all 7 time-slots are assigned to a single smart appliance the Zigbee link delay drop down to 0.015 and $0.043 \mathrm{sec}$. This is the theoretical minimum delay.

The analysis is further extended to include effect of WiFi interference, resulting in unavailable channel for Zigbee transmission. In Figure 3, the y-axis extends to 15 second, which is the maximum delay for AMI function (Table I). The results indicate that at $20 \%$ probability of clear channel assessment and 1 assigned GTS, the delay is above the maximum allowed (point A on the graph). In the case of 1 GTS, even at $99 \%$ probability of clear channel assessment the delay bound is 3.6 seconds (point C), which is unsatisfactory for Demand Response. Consistent with Figure 2, the delay drops sharply as the number of assigned GTS slots are increased (slope between points $\mathrm{C}$ and $\mathrm{D}$ ). The minimum theoretical environment that satisfies AMI requirements of $15 \mathrm{sec}$ delay in the case of 7 GTS assignment is around $2.5 \%$ clear channel probability (point F). The last two points shown on the graph, points B and E, represent the boundary for probability of clear channel assessment parameter after which performance degrades rapidly. Table IV presents summary of the the points of interest. Futhermore, the theoretical operational areas for AMI and DR functions are highlighted. For AMI most of the regions are 


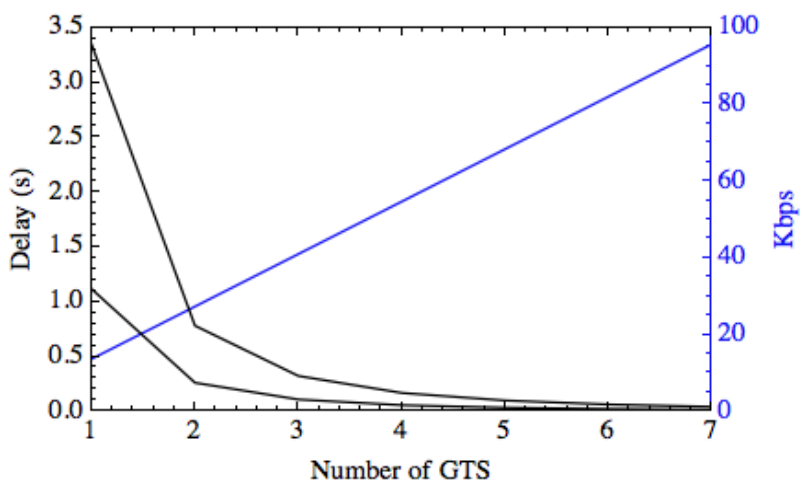

Fig. 2. Maximum, minimum delay and guranteed Kbps per client as a function of number of assigned GTS per client.

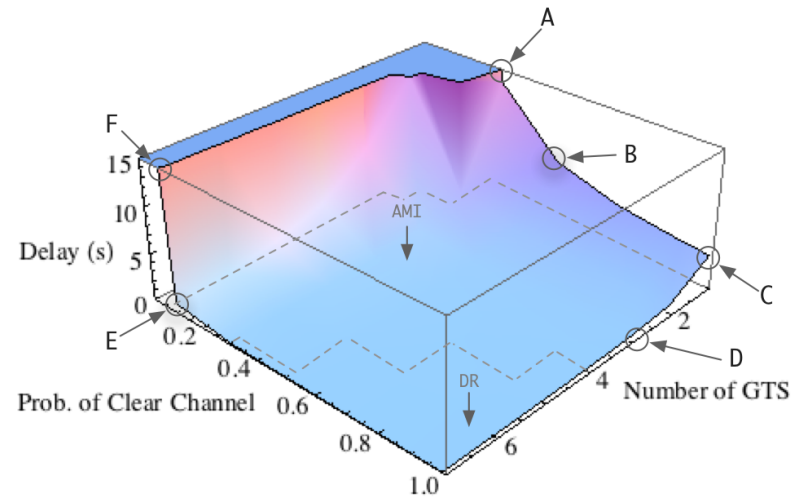

Fig. 3. Maximum delay and guranteed Kbps per client as a funciton of number of assigned GTS per client, and probabilty of clear channel accesment by the client. included, with the exception of probability of clear channel assessment under 0.1 for the case of 3 GTS slots or greater, and 0.2 for 2 GTS or fewer. The DR operational region is bounded by the south corner of the graph and the points (presented as $\{$ GTS, probability of CCA $\}$ coordinates): $\{4,0.99\},\{5$, $0.7\},\{6,0.5\}$ and $\{7,0.3\}$. Any area outside of this region theoretically does not satisfy the DR's functional requirements.

\begin{tabular}{llll}
\hline Point & Prob. of CCA & GTS & Delay \\
\hline A & 0.2 & 1 & $15 \mathrm{~s}$ (greater than) \\
B & 0.5 & 1 & $7.1 \mathrm{~s}$ \\
C & 0.99 & 1 & $3.6 \mathrm{~s}$ \\
D & 0.99 & 3 & $0.4 \mathrm{~s}$ \\
E & 0.1 & 7 & $1.02 \mathrm{~s}$ \\
F & 0.1 & 7 & $15 \mathrm{~s}$ (greater than) \\
\hline \multicolumn{5}{r}{ TABLE IV }
\end{tabular}

SUMMARY OF BOUNDRY POINTS OF INTEREST CALCULATED FROM THE MODEL.

\section{CONCLUSION}

In this paper, we presented a performance model of Smart Energy Profile protocol in regards to Advanced Meter Infrastructure (AMI) and Demand Response (DR) function of Smart Grid. A Network Calculus formulation was used to evaluate the performance of Zigbee based Home Area Network in Guaranteed Time Slot mode of operation. The model accounted for number of GTS assignments per client and interference caused by co-located WiFi networks. The outputs of the model are guaranteed throughput per time slot, and minimum and maximum delay. Results show that each input parameter has significant impact on the protocol's performance.

\section{REFERENCES}

[1] U. S. D. O. Energy, The Smart Grid: An Introduction, pp. 148, Sep. 2008.
[2] J. F. Editor, Draft Report on the NIST Framework and Roadmap for Smart Grid Interoperability Standards, pp. 1227, Feb. 2012.

[3] NIST, Report to NIST on the Smart Grid Interoperability Standards Roadmap.

[4] D. O. Energy, DEPARTMENT OF ENERGYCOMMUNICATIONS REQUIREMENTS OF SMART GRID TECHNOLOGIES, pp. 169, Oct. 2010.

[5] [M. Balakrishnan, Smart Energy Solutions for Home Area Networks and Grid-End Applications, pp. 17, Jan. 2012.

[6] P. Jokar, Model-based Intrusion Detection for Home Area Networks in Smart Grids, pp. 19, Apr. 2012.

[7] Z. Allience, ZigBee and Wireless Radio Frequency Coexistence, pp. 115, Apr. 2011.

[8] P. Yi, A. Iwayemi, and C. Zhou, Developing ZigBee Deployment Guideline Under WiFi Interference for Smart Grid Applications, IEEE Trans. Smart Grid, vol. 2, no. 1, pp. 110120.

[9] [G. Anastasi, M. Conti, and M. Di Francesco, The MAC Unreliability Problem in IEEE 802.15.4 Wireless Sensor Networks, pp. 18, Jul. 2009.

[10] F. Chen, N. Wang, R. German, and F. Dressler, Simulation study of IEEE 802.15.4 LR-WPAN for industrial applications, Wirel. Commun. Mob. Comput., pp. n/an/a, 2009.

[11] A. ZigBee, ZigBee Specification, pp. 1604, Jan. 2008

[12] A. ZigBee, SEP 2.0 Application Specification 0.9 Document, pp. 1352, Jul. 2012.

[13] K. Holbrook, Smart Energy Profile version 2.0 Technical Requirements Document, pp. 1112, Aug. 2012.

bibitemIEEEhowto:kopka A. Koubaa, M. Alves, and E. Tovar, GTS Allocation Analysis in IEEE 802.15.4 for Real-Time Wireless Sensor Networks, pp. 18, Mar. 2006

[14] G. Thonet, A.-J. Patrick, and P. Coller, ZigBee WiFi Coexistence, pp. 138, Apr. 2008.

[15] C.-J. M. Liang, N. B. Priyantha, J. Liu, and A. Terzis, Surviving Wi-Fi Interference in Low Power ZigBee Networks, pp. 114, Sep. 2010.

[16] A. Ghosh, R. Jana, V. Ramaswami, J. Rowland, and N. K. I. 2. P. I. Shankaranarayanan, Modeling and characterization of large-scale Wi-Fi traffic in public hot-spots, presented at the INFOCOM, 2011 Proceedings IEEE.

[17] W. Yang, X. Wang, and J.-P. Linnartz, A Coexistance Model of IEEE 802.15.4 and IEEE $802.11 \mathrm{~b} / \mathrm{g}$, ieeexplore.iee.org. 